\title{
Internal Versus External Effector and Transcription Factor Gene Pairs Differ in Their Relative Chromosomal Position in Escherichia coli
}

\author{
Sarath Chandra Janga ${ }^{1 *}$, Heladia Salgado ${ }^{1}$, Julio Collado-Vides ${ }^{1}$ \\ and Agustino Martínez-Antonio ${ }^{2 *}$
}

\author{
${ }^{1}$ Programa de Genómica \\ Computacional. Centro de \\ Ciencias Genómicas \\ Universidad Nacional \\ Autónoma de México \\ Cuernavaca, Morelos \\ 62100, México
}

${ }^{2}$ INSERM U511

Immunobiologie Cellulaire et Moléculaire des Infections Parasitaires, Université Pierre et Marie Curie, CHU-Pitié Salpetrière, 75013 Paris, France

${ }^{*}$ Corresponding authors

\begin{abstract}
Transcription factors (TFs) play an important role in the genetic regulation of transcription in response to internal and external cellular stimuli. However, little is known about their functional and dynamic aspects on a large scale, even in a well-studied bacterium like Escherichia coli. To understand the regulatory dynamics and to improve our knowledge about how TFs respond to endogenous and exogenous signals in this simple bacterium model, we previously proposed that TFs can be classified into three classes, depending on how they sense their allosteric or equivalent metabolite: external class, internal class, and hybrid sensing class. Classification of these groups was done without considering the relative chromosomal positions of the TFs and their corresponding effector genes. Here, we analyze the genome organization of the genetic components of these sensing systems, using the classification described earlier. We report the chromosomal proximity of transcription factors and their effector genes to sense periplasmic signals or transported metabolites (i.e. transcriptional sensing systems from the external class) in contrast to the components for sensing internally synthesized metabolites, which tend to be distant on the chromosome. We strengthen our finding that external sensing genetic machinery behaves like chromosomal modules of regulation to respond rapidly to variations in external conditions through co-expression of their genetic components, which is corroborated with microarray data for E. coli. Furthermore, we show several lines of evidence supporting the need for the coordinated activity of external sensing systems in contrast to that of internal sensing machinery, which can explain their close chromosomal organization. The observed functional correlation between the chromosomal organization and the genetic machinery for environmental sensing should contribute to our understanding of the logical functioning and evolution of the transcriptional regulatory networks in bacteria.
\end{abstract}

(C) 2007 Elsevier Ltd. All rights reserved.

Keywords: Escherichia coli; transcription factors; allosteric interactions; cell sensing; regulatory-metabolic network
Present address: A. Martínez-Antonio, Departamento de Ingeniería Genética, Centro de Investigación y de Estudios Avanzados, Campus Guanajuato, C.P. 36500, Irapuato, México.

Abbreviations used: TF, transcription factor; TU, transcription unit.

E-mail addresses of the corresponding authors: sarath@ccg.unam.mx; agustinom@ira.cinvestav.mx

\section{Introduction}

Organisms need to monitor environmental conditions constantly in order to respond correctly to changes. Physicochemical signals are recognized by the cell as messengers of environmental composition as well as its metabolic state. ${ }^{1-3}$ Bacterial cells have developed diverse strategies to sense these environmental signals, including signal transduction in chemotaxis, ${ }^{4,5}$ serine/threonine kinases and phosphatases, $^{6-8}$ riboswitches, ${ }^{9}$ etc. However, the 


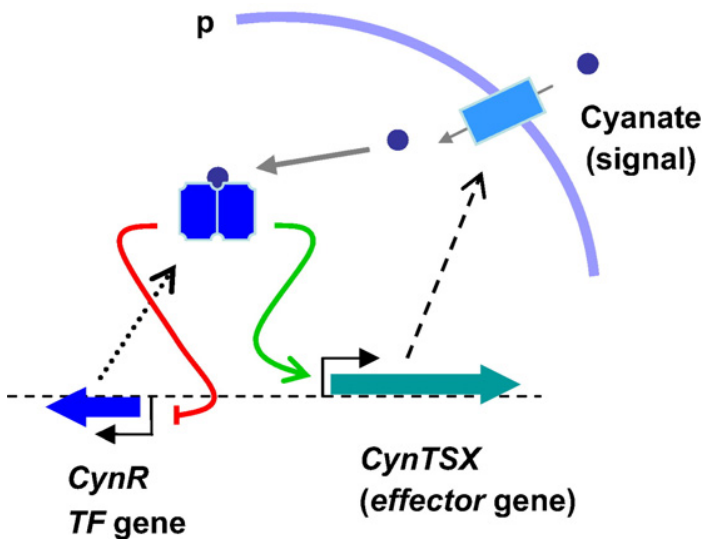

Figure 1. An example showing the mechanism of action of a transcriptional sensing system observed in $E$. coli. The effector gene $c y n X$ (operon cynTXS) encodes for a transport system for the uptake of cyanate from the surrounding milieu ( $p$, periplasm) and is used as a nitrogen source. CynR TF regulates its own transcription negatively in a cyanate-independent manner; however, in the presence of an adequate concentration of the metabolic signal (cyanate), CynR modifies its binding conformation (and presumably bends the DNA) in the DNA-regulatory region between $c y n T X S$ and $c y n R$ operons, activating the transcription of the former. ${ }^{39}$

genetic machinery in charge of regulating the expression of genes can respond directly to changes in the presence of these signals through the regulatory activity of transcription factors (TFs). Indeed, the binding of TFs to specific allosteric signals determines their active or inactive conformation and their affinity for interaction with specific sequences on DNA in the regulatory regions of transcription units or with the rest of the transcriptional machinery. ${ }^{10}$ Thus, transcription factors can be considered as molecular switches that respond to specific input signals and give transcriptional responses as output. ${ }^{11}$ On the other hand, studies in Escherichia coli have shown the influence of DNA supercoiling in gene transcription, ${ }^{12,13}$ and the perception of a 3-D spatial structure of the chromosome correlated with patterns of gene expression. ${ }^{14,15}$ Using statistical analysis and gene expression data, it was possible to observe functional associations among neighboring transcription units (TU) where, in most cases, a TU coding for a TF was found to be regulating a contiguous non-TF coding $\mathrm{TU}, 16,17$ and these patterns seem to be conserved in bacteria. ${ }^{18}$ In addition, co-regulation of divergent and tandem sets of genes is a common theme in E. coli but not in the yeast Saccharomyces cerevisiae. ${ }^{19}$ Thus, it seems that chromosomal organization of genes might have an important role in the proper functioning of the cell. In this work, we analyze the chromosomal organization of TFs and their effector genes using a previously developed classification of TFs in E. coli that was based on the cellular location of signals and direct-binding metabolites that TFs detect, specifically, whether such signals are of extracellular, intracellular or a combination of both origins. ${ }^{11,20}$ The findings reported here should enhance our understanding of the chromosomal order and functional organization of the genetic components for internal and external sensing machinery in bacteria, and provide insights to develop strategies for identifying regulatory elements in other organisms.

\section{Results and Discussion}

\section{TF and effector genes for exogenous sensing tend to be proximal on the $E$. coli chromosome}

The basic unit of sensing is constituted by TF and effector genes; the former encode for a TF sensing the effector signal produced or obtained by the product of the second gene, as shown in Figure 1.2,20 The main characteristics of the subclasses of the genetic sensing machinery in E. coli are described in Table 1

Table 1. Main properties of sensing classes identified in E. coli

\begin{tabular}{|c|c|c|}
\hline Sensing class & TF gene product function & Effector gene product function \\
\hline $\begin{array}{l}\text { E-TC (two-component systems } \\
\text { of signal transduction) }\end{array}$ & $\begin{array}{l}\text { Cytoplasmic soluble proteins, response } \\
\text { regulators to messages detected by } \\
\text { periplasmic sensor components }\end{array}$ & $\begin{array}{l}\text { Mostly periplasmic sensor protein to } \\
\text { monitor external signals }\end{array}$ \\
\hline $\begin{array}{l}\text { E-TM (sense transported } \\
\text { metabolites) }\end{array}$ & TFs binding transported metabolites & $\begin{array}{l}\text { Transport systems for transporting } \\
\text { metabolites from the surrounding milieu }\end{array}$ \\
\hline $\begin{array}{l}\text { H (sense synthesized } \\
\text { and transported } \\
\text { metabolites) }\end{array}$ & $\begin{array}{l}\text { TFs binding transported as well as } \\
\text { cytoplasmic synthesized metabolites }\end{array}$ & $\begin{array}{c}\text { Two kinds of signal genes: transport and enzyme proteins. } \\
\text { On the basis of whether the effector gene is an enzyme or } \\
\text { a transport protein, a hybrid system can be further } \\
\text { classified into HE or HT, respectively }\end{array}$ \\
\hline $\begin{array}{l}\text { I-SM (sense internally } \\
\text { synthesized metabolites) }\end{array}$ & $\begin{array}{l}\text { TFs binding metabolites } \\
\text { synthesized in the cytoplasm }\end{array}$ & Cytoplasmic enzyme proteins \\
\hline
\end{tabular}

The main properties of signal sensing classes identified in E. coli K12.Complete classification of the TFs into different classes of sensing based on evidence from the literature is available in RegulonDB. ${ }^{21}$ (The complete classification is also available as Supplementary Data.) 
(and see Supplementary Data) and a more complete discussion is presented elsewhere. ${ }^{20}$ In this work, we used information on TFs and effector genes with the experimental evidence gathered in the RegulonDB database. ${ }^{21}$ We obtained data for 84 TFs and 291 corresponding effector genes. This set of TFs corresponds to $29 \%$ of the roughly 297 predicted TFs in E. coli, ${ }^{22,23}$ and we added the TFs that are known to be part of two-component systems or whose function was annotated for $E$. coli. The final set consisted of 113 TFs covering around $38 \%$ of the total TF collection. It is important to note that the initial classification was done without considering the relative chromosomal positions of the TFs and their effector genes.

The distance on the chromosome between the TF and its corresponding effector gene(s) was measured as the minimum number of intervening genes separating them on either strand. We normalized this distance with the maximum distance, which is equal to half the number of total annotated proteincoding genes on this circular chromosome. Figure 2 shows a remarkable tendency for the TF-effector gene pairs in E-TC and E-TM categories to be located close to each other on the chromosome in contrast to that of the internal and hybrid classes of sensing. For the E-TC subclass, 26 (90\%) of the 29 predicted response regulators are at one or two genes of distance with respect to their corresponding effector genes (which encode for sensor proteins) and exclusively in a tandem orientation. ${ }^{24}$ These short distances are significant, because the largest operons in $E$. coli where genes are generally co-regulated comprise no more than 15 genes. Therefore, this chromosome arrangement for sensor and response regulators in two-component systems might be in agreement with the functional roles of these proteins working in concert as one unit, or as one TF with a functional resemblance to two proteins. ${ }^{25}$ The tandem arrangement might be the best way to achieve a concerted expression and translation, and to generate a timely response in order to minimize the cross-talk between these signal transduction systems (see below).

In the case of the E-TM subclass, the effector genes encode for transport systems, and $69 \%$ of TFs (20 of 29) were found to have all their effector genes within a chromosomal distance of 15 genes, and $90 \%$ have at least one effector gene within this short distance (see Figure 2(b)). As in twocomponent systems, the commonest arrangement in the E-TM subclass is that of tandem organization of TF-effector gene pairs, although some cases of divergent and convergent orientation were observed. The physical proximity of TF and the operons, encoding for the transporter genes might be because most of these transported metabolites are, in turn, catabolic substrates whose assimilation initiates the activity of specific pathways, genes involved in which are also encoded in these closely located operons. This arrangement of TFs and effector genes in close proximity permits them to react in concert to the stimulus of assimilatable metabolites in the surrounding milieu, as local genetic modules of regulation. These arrangement properties might be conserved in some groups of related bacteria, in the light of a study that revealed conservation of genes for transport functions encoded into catabolic operons in 26 bacterial genomes; all of them conserve one adjacent TF regulating the transcription of these operons, ${ }^{26}$ despite the poor conservation of the network components across all bacteria. ${ }^{27,28}$

\section{Genes encoding enzymes and TFs for synthesizing and sensing endogenous signals tend to be distant on the chromosome}

Contrary to two-component systems (E-TC) and E-TM for sensing transported metabolites, both of which are used mostly for extracellular sensing, the effector genes (enzymes) synthesizing metabolites in (a)

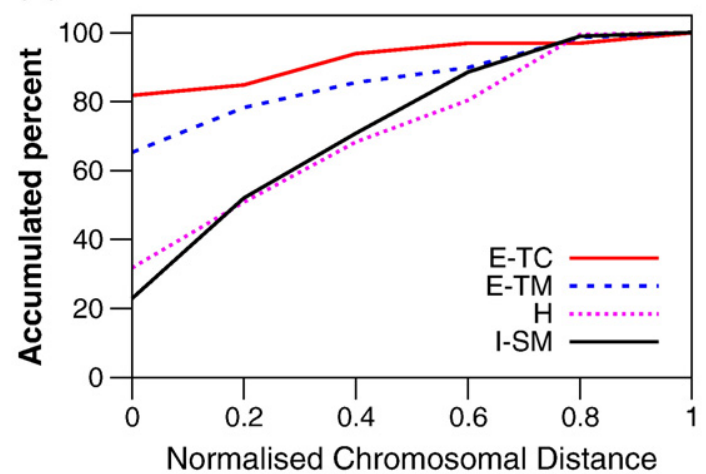

(b)

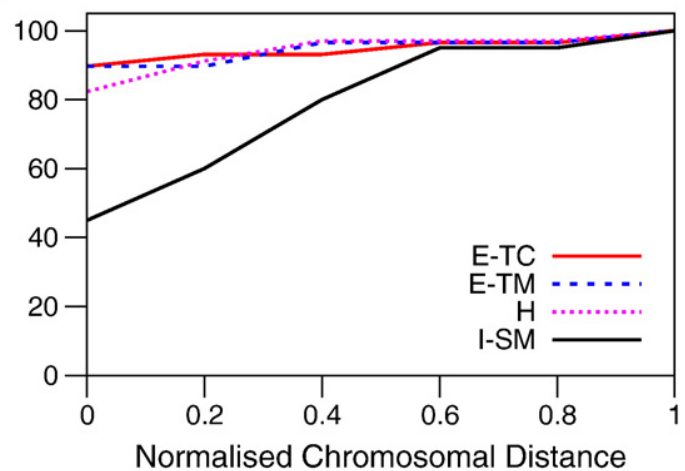

Figure 2. Normalized chromosomal distance (NCD) (defined as the fraction of intervening genes between the TF and effector gene compared to the maximum chromosomal distance, i.e. roughly half the protein-coding genes on a circular chromosome) between TF-effector genes in different sub-classes of sensing. (a) Accumulated percentage of TF-effector pairs as the chromosomal distance of their effector genes increases for each subclass of sensing. (b) Accumulated percentage of TF-effector pairs considering for each TF only the nearest effector gene when more than one effector gene has been reported (see Supplementary Data for details). 


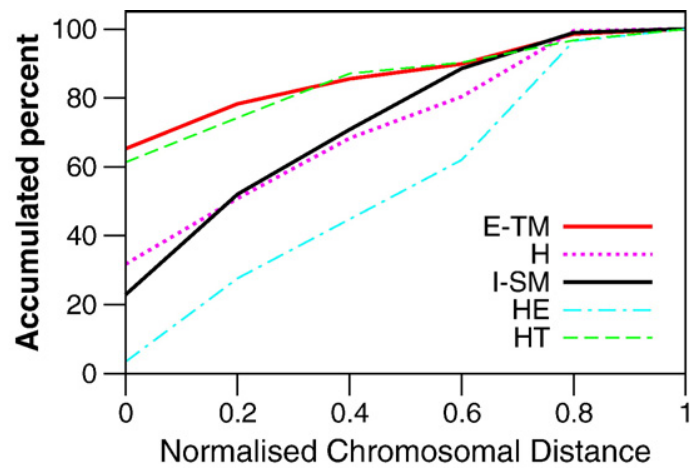

Figure 3. Accumulated percentage versus the normalized chromosomal distance (NCD) for TF-effector gene pairs in the hybrid class of sensing when the transporters and enzymes are considered as different sub-sets. If more than one transporter is associated with a given TF, the closest was considered, while for enzymes the farthest gene was considered. The H-transport accumulated percentage profile was found to resemble that of the E-TM, while the H-enzyme profile shows large chromosomal distances like those observed in the I-SM category.

the cytoplasm tend to be encoded distantly on the chromosome with respect to the TFs, which the synthesized metabolite is affecting. Only four of 20 TFs $(20 \%)$ have an effector gene at a distance of less than 15 genes (Figure 2). It might be that signals, TFs or both components are at high concentrations or that diffusion or dilution is not a limiting factor for the interaction of these cellular components in the bacterial cytoplasmic space. It is known that genes encoding for enzymes in small metabolite synthesis in E. coli tend to be close together on the chromosome when they are less than four steps away in a metabolic pathway. ${ }^{29}$ Thus, it might be that the arrangement of genes encoding for enzymes in the same metabolic pathway is constrained to physical proximity on the chromosome but not in proximity to the TFs that influence their activity. This might be due to the need for these genes to be expressed in a "just-in-time" fashion, as demonstrated for amino acid biosynthesis pathways. ${ }^{30}$

\section{TF and effector pairs from the hybrid class exhibit chromosomal distances intermediate between those of the internal and external sensing machinery}

It is interesting to note from Figure 2(b) that the TFs from the hybrid class of sensing, which are controlled by mixed signals of both internal and external origin, show a profile of chromosomal distances intermediate between those of the internal and external subclasses. Since the hybrid class of sensing includes genes encoding transport systems and enzymes, it was interesting to analyze them independently. To understand if the chromosomal distance of TFs from hybrid class with respect to their effector genes varies in each of these (transporters and enzymes) effector gene categories, we first identified the effector genes encoding for proteins involved in transport and enzymatic functions and then obtained the chromosomal distances (closest effector gene for transporters and the farthest for enzyme coding genes was used if more than one effector gene was annotated for a given TF of each type) with respect to their TFs. We found that, while the transporter-encoding genes show an accumulated frequency curve similar to that of systems from E-TM category, enzyme-coding genes present a distance profile close to that of the I-SM subclass of sensing, as shown in Figure 3. However, it is not clear why bacterial cells employ two strategies to obtain these same metabolites; transporting them from the surrounding milieu and synthesizing them in the cytoplasm. An important starting point to understand these variations could be to determine in which part of the metabolism these metabolites are being produced or incorporated and to try to understand the hierarchy in the decision making for prioritizing the transport of available metabolites in the surrounding milieu and their intracellular synthesis.

\section{Chromosomal distances between TF and effector genes sensing exogenous signals tend to be significantly shorter than those of the other sensing classes}

In order to test the statistical significance of the trend observed above, i.e TFs for sensing exogenous signals to be in close proximity with their effector genes in contrast to that of the internal sensing TFs, we performed a comparison of the average distances observed between TF and effector genes in each class of sensing against those constructed randomly. Briefly, the distances observed in each class of sensing were compared against that of the average distances seen in randomly generated set of 10,000 TF-effector gene combinations for each subclass of sensing (see Materials and Methods). As shown in Table 2, we found that the tendency for TFs in external sensing classes E-TM and E-TC to be in close proximity to their effector genes was highly significant $\left(P\right.$-values $<10^{-14}$ ), unlike in I-SM and hybrid classes, where the distances observed seem close to those in randomly generated samples. This is evidenced from the Z-scores in these two categories, which are greater than three negative deviations,

Table 2. Statistical significance of the average chromosomal distances observed in each class of sensing in comparison to the distances seen in a randomly generated collection of TF-effector gene pairs

\begin{tabular}{lccc}
\hline $\begin{array}{l}\text { Sensing } \\
\text { class }\end{array}$ & $\begin{array}{c}\text { Average chromosomal } \\
\text { distance (observed) }\end{array}$ & Z-score & $P$-value \\
\hline I-SM & 902 & -1.633 & 0.1025 \\
H & 896 & -2.903 & 0.0037 \\
E-TC & 217 & -7.966 & $<10^{-14}$ \\
E-TM & 378 & -8.586 & $<10^{-17}$ \\
\hline
\end{tabular}

The average chromosomal distance for each class of sensing stands for the average number of intervening genes observed between TF and effector genes is rounded to the nearest integer. 
indicating no special preference for shorter distances. It is clear from Table 2 that TFs from the hybrid class of sensing are slightly closer to their signal genes in comparison to TFs from the I-SM category. This is interesting, because these systems, which are composed of effector genes that can sense signals of both internal (enzymes) and external (transporters) origin, might have them organized differently, as observed above, thereby reducing the tendency for shorter distances as seen in external sensing systems.

\section{TF and effector genes sensing exogenous signals tend to be co-expressed}

To investigate further if the chromosomal proximity of TFs and effector genes involved in extracellular sensing is due to the functional need for these systems to co-ordinate their expression, we used the data of gene expression from microarray experiments in E. coli as described in Materials and Methods. In Figure 4, we show the proportion of TFeffector gene pairs in each class of sensing as the Pearson correlation increases. It is clear from the graph that the sensing systems from E-TM and E-TC categories are highly co-expressed compared to the I-SM category. The correlation of expression between TF and effector genes in the E-TC category is worth mentioning, because these sensing systems are generally known to occur in close neighborhood on the chromosome and often in operons, and we found that they are always highly correlated in their transcription.

To address the dependence of TF-effector gene pair chromosomal distance on their pattern of coexpression in the different classes of sensing, the correlation of expression for each sensing system was plotted as shown in Figure 5. There is a clear

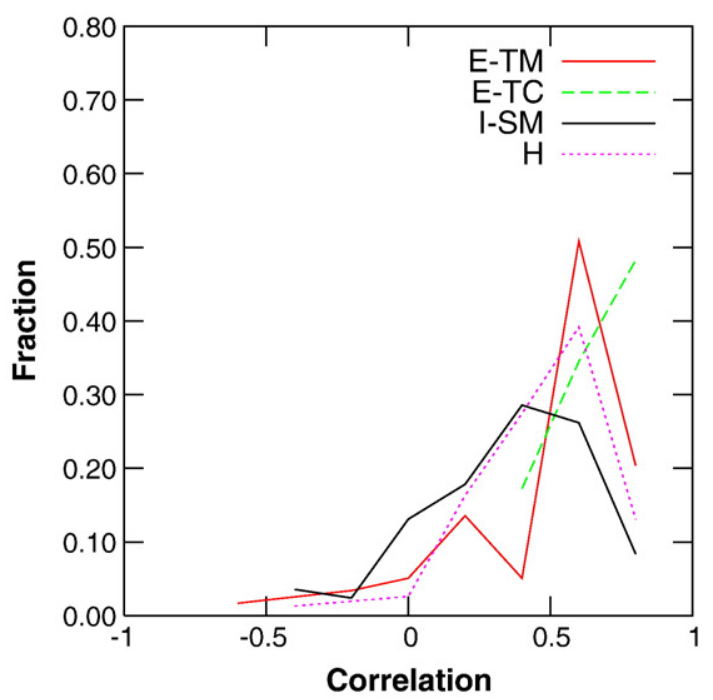

Figure 4. The proportion of TF-effector gene pairs in each class of sensing with a given correlation of expression in microarray experiments as the Pearson correlation increases. The data are binned in intervals of 0.2 on the $X$-axis. tendency for sensing systems from the E-TM and ETC categories to be highly correlated even when the chromosomal distances of the TF-effector gene pairs are not short, unlike those from the I-SM and $\mathrm{H}$ categories, which are scattered widely on this plot of chromosomal distance versus correlation of expression. Despite the strong tendency for chromosomally close TF-effector gene pairs to be highly coexpressed in both the E-TM and E-TC categories; there were exceptional cases in the E-TM category where the sensing systems with short distances are poorly correlated in transcription. In Table 3 we show these cases from the E-TM category where the observed Pearson correlation was $<0.4$ despite the distances being short. It is possible that these sensing systems are poorly expressed in all the conditions of the microarray experiments analyzed and hence have poor correlation. An alternative explanation could be that our current classification of TFs is not complete due to the lack of relevant information about the correct or additional effector genes for some of these TFs. Irrespective of the reasons that might best explain the poor correlation of some of these cases, it is interesting to explore them in greater detail. It would also be of interest to explore in the external classes of sensing those cases where the sensing systems are highly correlated in expression despite being far apart on the chromosome. One can speculate that there might be intervening signals that could be responsible for the rapid response and high co-expression of such TF-signal gene pairs. However, an extensive analysis is needed to study and understand these cases.

\section{Chromosomal positioning, transcriptional and physical protein-protein interactions might be the strategies used by two-component systems for coordinating their activity}

Since two-component systems use trans-phosphorylation as a common process for signal transduction between sensor components and response regulators, there is a high possibility for cross-talk and interference in the processing of signal messages between these pairs of systems. ${ }^{24}$ As we describe above, the genes encoding for two-component systems tend to be contiguous pairs on the chromosome; hence, it was interesting to understand the way in which these systems are regulated and how they interact at the level of protein-protein interactions. In Figure 6(a) we show the currently available information on the transcriptional regulation of twocomponent genes in E. coli. ${ }^{21}$ It was possible to obtain regulatory information for 16 response regulators and for 12 pairs of sensor-response genes. It can be seen that there is coordination in the regulation of these pairs of genes, as in seven cases the response regulator is directly regulating the corresponding sensor component and in four other pairs, genes are being co-regulated by other TFs; narX/narL (nitrate and nitrite regulation) and $\mathrm{dcuR/dcuS} \mathrm{(di-carboxylic} \mathrm{acids} \mathrm{sensing)} \mathrm{are} \mathrm{being}$ 


\section{E-TM}

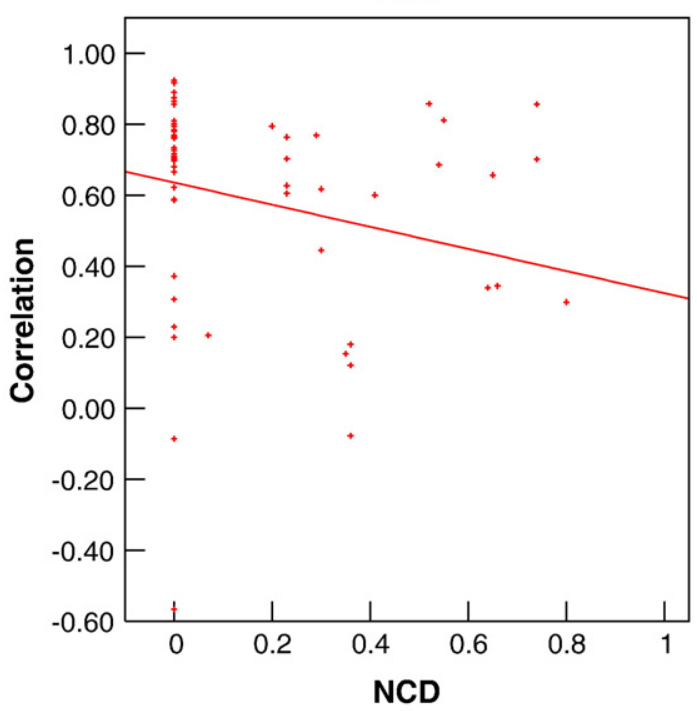

I-SM

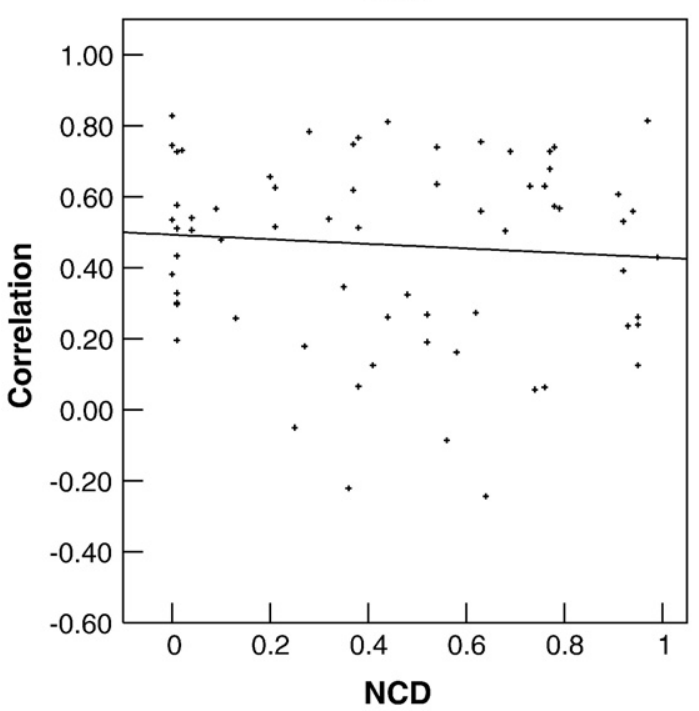

E-TC

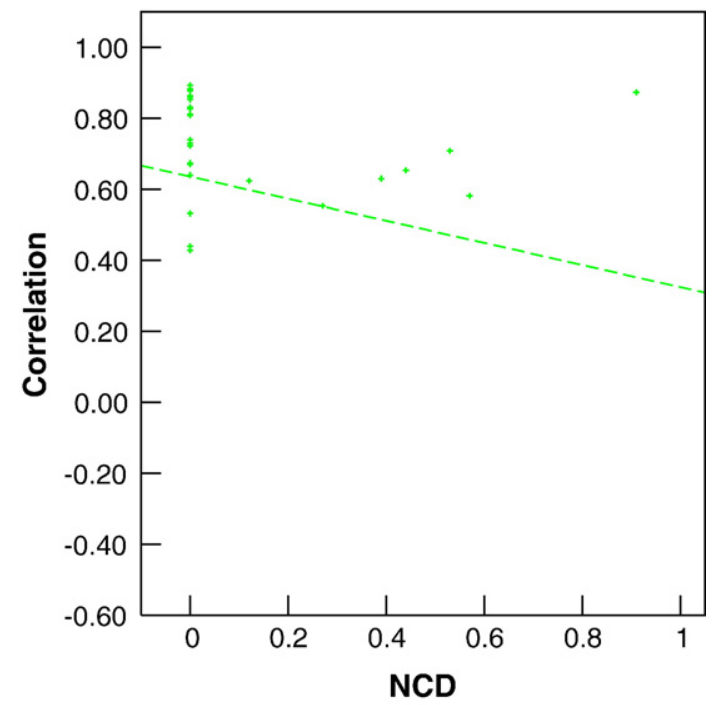

H

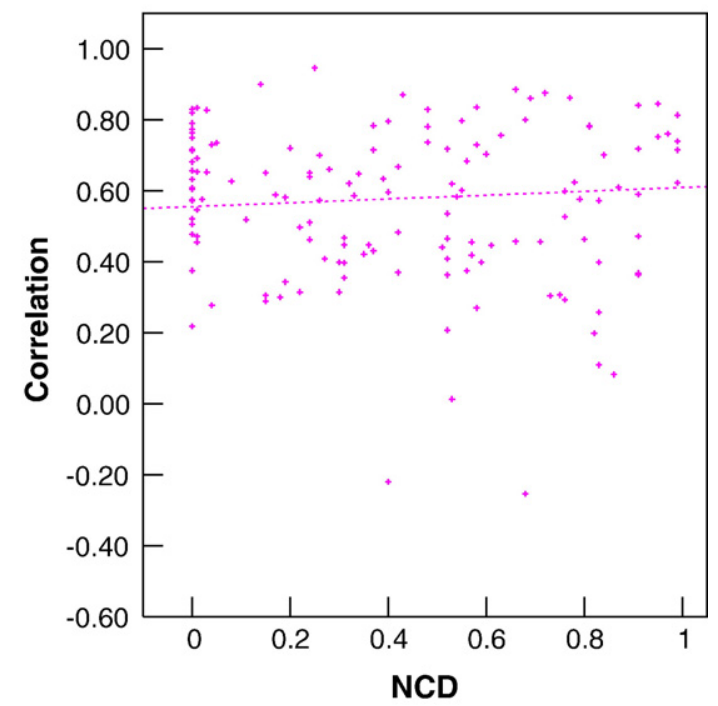

Figure 5. Normalized chromosomal distance (NCD) versus Pearson correlation $(r)$ for TF-effector gene pairs from different classes of sensing. The data are fit to a straight line using the median values of $r$ from each 0.1 interval of NCD to show the trend. Only the intervals in which at least three pairs occur were considered for calculating the median. TF-effector gene pairs from sensing classes, E-TM and E-TC, which sense signals of exogenous origin, can be seen to be highly co-expressed when they are chromosomally close. On the contrary, TF-effector gene pairs from the I-SM class do not show any special preference on the correlation axis ( $Y$-axis) with the variation of distance, showing that strong coexpression is not a prerequisite for the functioning of these systems.

co-regulated by ModE (molybdate-dependent regulator) and FNR (fumarate-nitrate regulator); zraR/zraS $\left(\mathrm{Zn}^{2+}\right.$ and $\mathrm{Pb}^{2+}$ sensing) and ompR/ envZ (osmotic pressure sensing) are regulated together by CRP (catabolic repressor protein), and the last pair is regulated also by the integration host factor (IHF). At the level of protein -protein interactions, we found interactions among $82 \%$ of the total pairs of genes from two component systems from the APID database. ${ }^{31}$ Protein-protein interactions included the pairs ArcA/ArcB (aerobicrespiration regulation), NarP/NarQ (nitrate and nitrite regulation) and the predicted UvrY/BarA system. These two-component systems are the only cases where constituent genes are separated on the chromosome. Thus, even when these pairs of genes are not encoded together on the chromosome they co-ordinate their activity, as is evidenced by gene coexpression and protein-protein interaction analysis, which might help to reduce the cross-talk. On the other hand, protein-protein interaction does not seem to be a rule for TFs and effector proteins in the other sensing classes as shown in Figure 6(b), where we represent the proteins interacting with TFs of other classes in the form of a network. Thus, it seems that in order to minimize the cross-talk between two-component systems, bacteria have developed the strategy of close chromosomal positioning of the 
Table 3. TF-effector gene pairs from the E-TM class of sensing, which are poorly correlated despite being chromosomally close

\begin{tabular}{lc}
\hline TF/effector gene & Pearson correlation \\
\hline srlR/srlB & 0.31 \\
cynR/cynX & -0.09 \\
gutM/srlB & 0.37 \\
caiF/caiT & 0.20 \\
zntR/zntA & 0.21 \\
lacI/lacy & 0.23 \\
lacI/lacZ & -0.56 \\
\hline
\end{tabular}

components, co-regulated expression and physical interaction of the protein products.

\section{Transcriptional regulation of effector genes by their respective TFs is predominant in the E-TM class of sensing}

In order to understand and quantitatively analyze the transcriptional regulation of the effector gene by the transcription factor whose activity is governed by it, we used the currently available data on transcriptional regulation in $E$. coli. ${ }^{21}$ We asked which class of sensing effector genes is controlled transcriptionally by their TFs. We found that the majority $(>60 \%)$ of the effector genes in the E-TM category are regulated by their corresponding TFs, suggesting that transcriptional regulation of the effector gene could be an important reason for these systems to be close together on the chromosome. On the contrary, effector genes from I-SM sensing systems for which transcriptional regulatory interaction data could be obtained were found to be regulated less than $30 \%$ of the time. In the case of the hybrid category, less than $40 \%$ of effector genes were found to be regulated by their corresponding TFs (see Supplementary Data for a complete set of TFs known to be regulating their effector genes in each class of sensing).

\section{Conclusions}

In this work, we demonstrate that TFs that sense signals of extracellular origin, defined as external sensing $\mathrm{TFs}^{20}$ which could be either response regulators to messages detected by periplasmic sensor components or those that can sense and bind to transported metabolites from the surrounding milieu, are organized on the chromosome in close neighborhood with their effector genes. We show evidence from microarray gene co-expression profiles, transcriptional regulatory and proteinprotein interactions such that an organization is specially emphasized in two-component systems, possibly to minimize the noise in response to variations in the external environment and to avoid cross-talk between these systems. It is interesting to speculate that the chromosomal proximity of TFs and effector genes observed for external sensing machinery might be limited to prokaryotic organisms and may not be extendable literally to eukaryotes cells due to the fact that transcription and translation are coupled in prokaryotes, rendering high local concentrations of the TF-effector, so that efficient regulation can occur. On the other hand, this might not be the case in eukaryotic cells, where transcription and translation occur in different compartments. However, even in eukaryotic cells there is evidence of "chromosomal territories" where gene co-expression is observed. ${ }^{32,33}$ In this way, even in complex organisms, it is possible that the simplest way to co-express genes is to organize them close together on the chromosome. Such a close organization can reduce problems caused by low copy numbers of TFs, and minimize uncertainties caused by stochastic events and problems due to diffusion caused by steep concentration gradients, thereby aiding an efficient and co-ordinated functioning of TF-effector genes in external sensing systems. An additional advantage for such a proximal organization of sensing systems on a prokaryotic chromosome could be the ease with which they can be transferred horizontally across genomes that thrive in the same environment. ${ }^{34}$ This is especially important and useful if the organisms depend on the availability of the same substrates in the environment, because many of the external sensing systems are involved in the transport of metabolites from the surrounding milieu, which would require a similar kind of machinery for a given kind of metabolite.

The analysis of extra- and intracellular sensing machinery in terms of chromosomal organization described here might contribute to our understanding of the logic of the cellular regulatory networks in bacteria. Disperse TFs responding to effector signals generated intracellularly versus a localized set of genes coding for TFs and their effector genes designed to sense fluctuant and specific exogenous signals. It is not known how this arrangement influences the topology of the regulatory networks. However, it is possible to infer from these observations that the scale-free nature of the regulatory networks could be explained in a biological context due to the presence of these local modules of regulation of external sensing category and global modules of regulation due to the internal sensing TFs. ${ }^{35,36}$

\section{Materials and Methods}

\section{Calculating the significance of chromosomal proximity of TF-effector genes}

To test the significance of chromosomal proximity of TFeffector genes in external sensing classes compared to those observed in the internal and hybrid classes of sensing, we first calculated the average chromosomal distance in each class of sensing and then compared that with the average chromosomal distance seen in a collection of 10,000 sets of randomly generated TF-effector genes for each sensing class. TF-effector gene pairs in each 
(a)
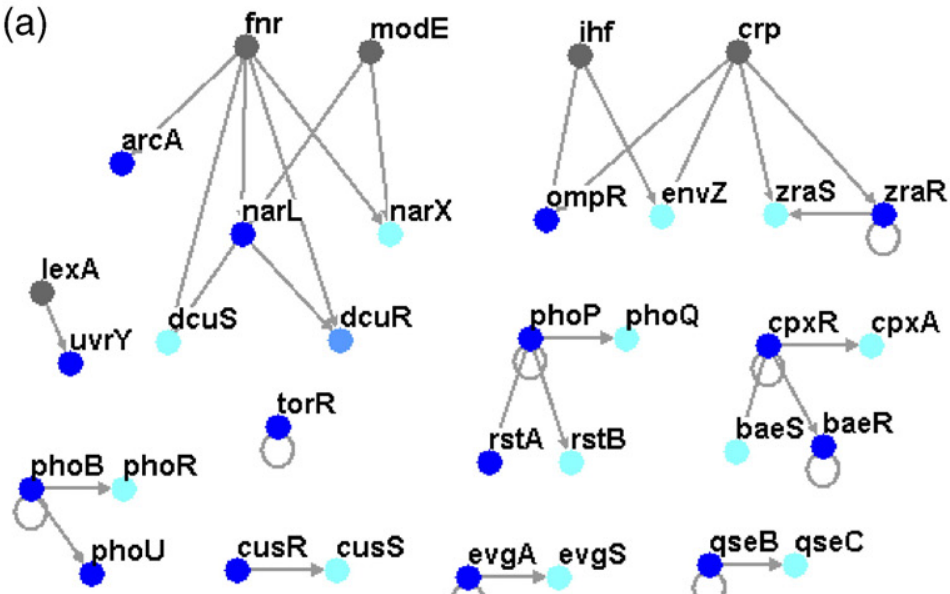

(b)
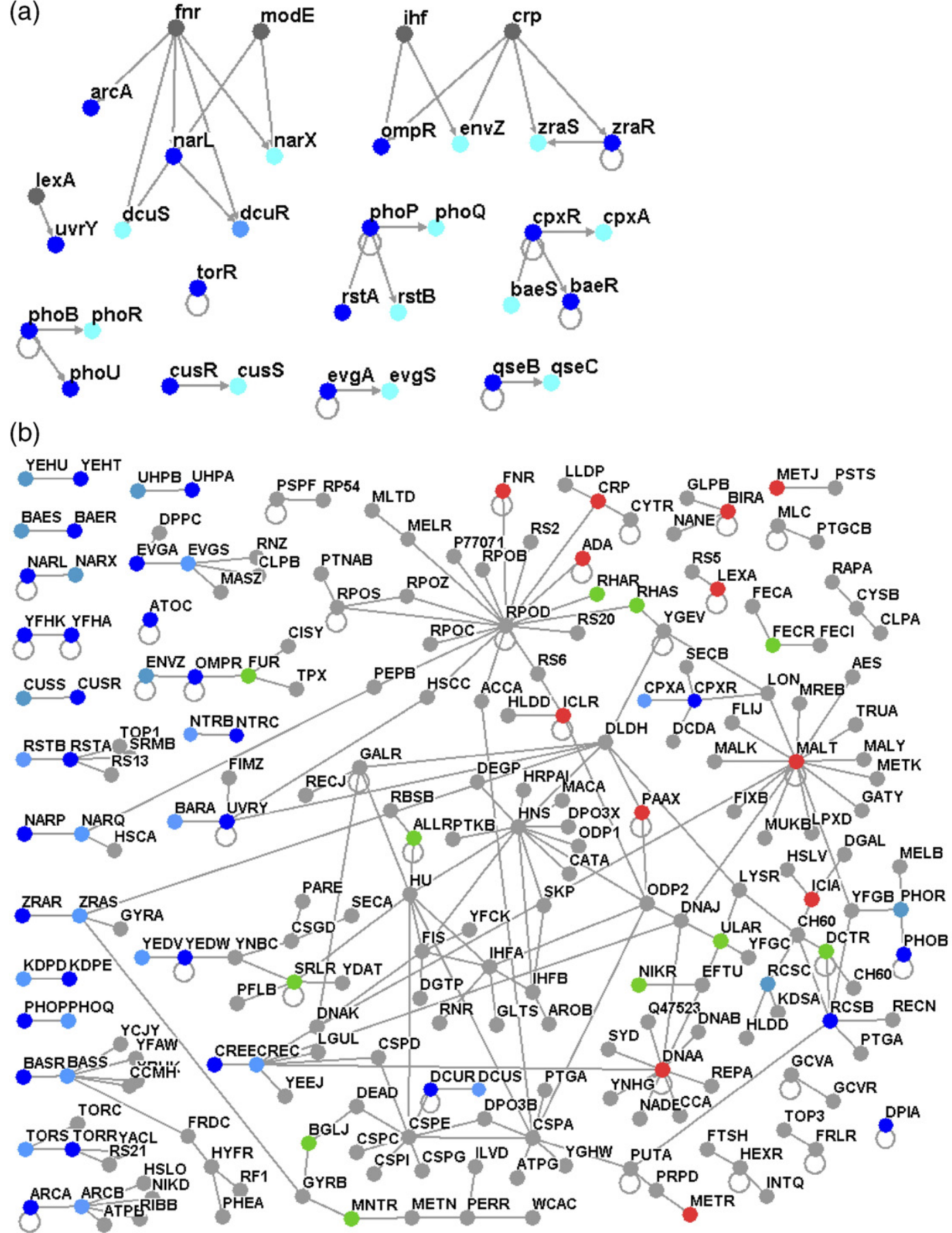

Figure 6. Transcriptional and protein-protein interaction networks in two-component systems. (a) Network showing the transcriptional regulation of genes encoding for two-component systems and interactions within themselves. Response regulators and sensor genes are represented in dark blue and light blue nodes, respectively. Arrows represent the sense of transcriptional regulation and loops represent auto-regulation of TFs. phoB/phoR, cusR/cusS, evgA/evgS, qseB/ qseC, cpxR/cpxA, phoP/phoQ and zraR/zraS are systems where the TF is known to regulate the expression of the sensor gene, while the systems $d c u R / d c u S$, narL/narX, ompR/envZ and zraR/zraS are known to be co-regulated by at least one TF (b) A network showing the known protein-protein interactions between TFs from different classes of sensing and other protein-coding genes in E. coli. ${ }^{31}$ Response regulators and sensor genes from E-TC systems are represented as shown in (a). TFs of I-SM are shown as nodes colored red, TFs of E-TM in green and in gray are shown for hybrid and DNA-bending TFs, as well as other proteins interacting physically with TFs not relevant to this study. Loops represent self-interactions. It is clear from this Figure that TFs from the E-TC sensing class frequently interact with their corresponding sensor proteins to form protein complexes, while it is not so common for TFs from other classes to interact with their respective effector genes. The network was constructed with the program Osprey. ${ }^{40}$ 
of the 10,000 random samples in each sensing class were obtained by randomly assigning an effector gene from the whole collection of effector genes known in any class of sensing. We made sure that the effector gene(s) associated with a given TF according to our classification were not assigned to the same TF in the process of randomization. The average chromosomal distance in a randomly generated collection of sensing classes was found to follow a gaussian distribution, so Z-scores were used to calculate $P$-values.

\section{Data of microarrays for $E$. coli}

We used the data of microarrays from 78 experiments obtained from the values reported by Price et al., ${ }^{37}$ as reported earlier. ${ }^{38}$ (See Supplementary Data for the complete data set). Briefly, to measure whether TFs and their corresponding effector genes have similar expression patterns over the set of experiments being analyzed, we employed the standard metric, Pearson linear correlation $(r)$ between the normalized log-ratios of the two genes. When calculating pairwise correlations, if one of the genes did not have expression values recorded in an experiment, that particular experiment was excluded from the calculation of $r$. A gene pair was considered to have some correlation only if both the genes under consideration had expression values recorded for at least ten different experiments.

\section{Acknowledgements}

We thank Ernesto Perez-Rueda and Gabriel Moreno-Hagelsieb for providing helpful suggestions to improve this work, and for their comments on previous versions of the manuscript. This work was supported, in part, by NIH grant RO1 GM 071962. A.M.-A. was supported by an INSERM fellowship for étranger chercheur. S.C.J. has been supported by grants given to J.C.V.

\section{Supplementary Data}

Supplementary data associated with this article can be found, in the online version, at doi:10.1016/ j.jmb.2007.01.019. Additional figures and tables related to this work can also be obtained from http: / /www. ccg.unam.mx/Computational_Genomics/TRNS/ CellSensing/. The regulatory sub-systems classified according to the location of signal metabolites used for the entire analysis can also be obtained from http:/ / www.ccg.unam.mx/Computational_Genomics/ regulondb/CellSensing/.

\section{References}

1. Camilli, A. \& Bassler, B. L. (2006). Bacterial smallmolecule signaling pathways. Science, 311, 1113-1116.

2. Wall, M. E., Hlavacek, W. S. \& Savageau, M. A. (2004). Design of gene circuits: lessons from bacteria. Nature Rev. Genet. 5, 34-42.
3. Shi, Y. \& Shi, Y. (2004). Metabolic enzymes and coenzymes in transcription-a direct link between metabolism and transcription? Trends Genet. 20, 445-452.

4. Baker, M. D., Wolanin, P. M., Stock \& J.B. (2006). Systems biology of bacterial chemotaxis. Curr. Opin. Microbiol. 9, 187-192.

5. Ben Jacob, E., Becker, I., Shapira, Y. \& Levine, H. (2004). Bacterial linguistic communication and social intelligence. Trends Microbiol. 12, 366-372.

6. Romling, U., Gomelsky, M. \& Galperin, M. Y. (2005). C-di-GMP: the dawning of a novel bacterial signalling system. Mol. Microbiol. 57, 629-639.

7. Galperin, M. Y. (2004). Bacterial signal transduction network in a genomic perspective. Environ. Microbiol. 6, 552-567.

8. Bijlsma, J. J. \& Groisman, E. A. (2003). Making informed decisions: regulatory interactions between two-component systems. Trends Microbiol. 11, 359-366.

9. Gilbert, S. D. \& Batey, R. T. (2006). Riboswitches: fold and function. Chem. Biol. 13, 805-807.

10. Browning, D. F. \& Busby, S. J. (2004). The regulation of bacterial transcription initiation. Nature Rev. Microbiol. 2, 57-65.

11. Seshasayee, A. S., Bertone, P., Fraser, G. M. \& Luscombe, N. M. (2006). Transcriptional regulatory networks in bacteria: from input signals to output responses. Curr. Opin. Microbiol. 9, 511-519.

12. Travers, A. \& Muskhelishvili, G. (2005). DNA supercoiling - a global transcriptional regulator for enterobacterial growth? Nature Rev. Microbiol. 3, 157-169.

13. Peter, B. J., Arsuaga, J., Breier, A. M., Khodursky, A. B., Brown, P. O. \& Cozzarelli, N. R. (2004). Genomic transcriptional response to loss of chromosomal supercoiling in Escherichia coli. Genome Biol. 5, R87.

14. Postow, L., Hardy, C. D., Arsuaga, J. \& Cozzarelli, N. R. (2004). Topological domain structure of the Escherichia coli chromosome. Genes Dev. 18, 1766-1779.

15. Jeong, K. S., Ahn, J. \& Khodursky, A. B. (2004). Spatial patterns of transcriptional activity in the chromosome of Escherichia coli. Genome Biol. 5, R86.

16. Warren, P. B. \& ten Wolde, P. R. (2004). Statistical analysis of the spatial distribution of operons in the transcriptional regulation network of Escherichia coli. J. Mol. Biol. 342, 1379-1390.

17. Guelzim, N., Bottani, S., Bourgine, P. \& Kepes, F. (2002). Topological and causal structure of the yeast transcriptional regulatory network. Nature Genet. 31, 60-63.

18. Korbel, J. O., Jensen, L. J., von Mering, C. \& Bork, P. (2004). Analysis of genomic context: prediction of functional associations from conserved bidirectionally transcribed gene pairs. Nature Biotechnol. 22, 911-917.

19. Hershberg, R., Yeger-Lotem, E. \& Margalit, H. (2005). Chromosomal organization is shaped by the transcription regulatory network. Trends Genet. 21, 138-142.

20. Martinez-Antonio, A., Janga, S. C., Salgado, H. \& Collado-Vides, J. (2006). Internal-sensing machinery directs the activity of the regulatory network in Escherichia coli. Trends Microbiol. 14, 22-27.

21. Salgado, H., Gama-Castro, S., Peralta-Gil, M., DiazPeredo, E., Sanchez-Solano, F., Santos-Zavaleta, A. et al. (2006). RegulonDB (version 5.0): Escherichia coli $\mathrm{K}-12$ transcriptional regulatory network, operon organization, and growth conditions. Nucl. Acids Res. 34, D394-D397.

22. Madan Babu, M. \& Teichmann, S. A. (2003). Evolution of transcription factors and the gene regulatory network in Escherichia coli. Nucl. Acids Res. 31, 1234-1244. 
23. Perez-Rueda, E. \& Collado-Vides, J. (2000). The repertoire of DNA-binding transcriptional regulators in Escherichia coli K-12. Nucl. Acids Res. 28, 1838-1847.

24. Yamamoto, K., Hirao, K., Oshima, T., Aiba, H. Utsumi, R. \& Ishihama, A. (2005). Functional characterization in vitro of all two-component signal transduction systems from Escherichia coli. J. Biol. Chem. 280, 1448-1456.

25. Ulrich, L. E., Koonin, E. V. \& Zhulin, I. B. (2005). Onecomponent systems dominate signal transduction in prokaryotes. Trends Microbiol. 13, 52-56.

26. Plantinga, T. H., van der Does, C. \& Driessen, A. J. (2004). Transporter's evolution and carbohydrate metabolic clusters. Trends Microbiol. 12, 4-7.

27. Lozada-Chavez, I., Janga, S. C. \& Collado-Vides, J. (2006). Bacterial regulatory networks are extremely flexible in evolution. Nucl. Acids Res. 34, 3434-3445.

28. Madan Babu, M., Teichmann, S. A. \& Aravind, L. (2006). Evolutionary dynamics of prokaryotic transcriptional regulatory networks. J. Mol. Biol. 358, 614-633.

29. Rison, S. C., Teichmann, S. A. \& Thornton, J. M. (2002). Homology, pathway distance and chromosomal localization of the small molecule metabolism enzymes in Escherichia coli. J. Mol. Biol. 318, 911-932.

30. Zaslaver, A., Mayo, A. E., Rosenberg, R., Bashkin, P., Sberro, H., Tsalyuk, M. et al. (2004). Just-in-time transcription program in metabolic pathways. Nature Genet. 36, 486-491.

31. Prieto, C. \& De Las Rivas, J. (2006). APID: agile protein interaction dataanalyzer. Nucl. Acids Res. 34, W298-W302.
32. Oliver, B., Parisi, M. \& Clark, D. (2002). Gene expression neighborhoods. J. Biol. 1, 4.

33. Belyakin, S. N., Christophides, G. K., Alekseyenko, A. A., Kriventseva, E. V., Belyaeva, E. S., Nanayev, R. A. et al. (2005). Genomic analysis of Drosophila chromosome underreplication reveals a link between replication control and transcriptional territories. Proc. Natl Acad. Sci. USA, 102, 8269-8274.

34. Pal, C., Papp, B. \& Lercher, M. J. (2005). Adaptive evolution of bacterial metabolic networks by horizontal gene transfer. Nature Genet. 37, 1372-1375.

35. Martinez-Antonio, A. \& Collado-Vides, J. (2003). Identifying global regulators in transcriptional regulatory networks in bacteria. Curr. Opin. Microbiol. 6, 482-489.

36. Vazquez, A., Dobrin, R., Sergi, D., Eckmann, J. P., Oltvai, Z. N. \& Barabasi, A. L. (2004). The topological relationship between the large-scale attributes and local interaction patterns of complex networks. Proc. Natl Acad. Sci. USA, 101, 17940-17945.

37. Price, M. N., Huang, K. H., Alm, E. J. \& Arkin, A. P. (2005). A novel method for accurate operon predictions in all sequenced prokaryotes. Nucl. Acids Res. 33, 880-892.

38. Janga, S. C., Lamboy, W. F., Huerta, A. M. \& MorenoHagelsieb, G. (2006). The distinctive signatures of promoter regions and operon junctions across prokaryotes. Nucl. Acids Res. 34, 3980-3987.

39. Lamblin, A. F. \& Fuchs, J. A. (1994). Functional analysis of the Escherichia coli K-12 cyn operon transcriptional regulation. J. Bacteriol. 176, 6613-6622.

40. Breitkreutz, B. J., Stark, C. \& Tyers, M. (2003). Osprey: a network visualization system. Genome Biol. 4, R22.

Edited by J. Karn

(Received 7 November 2006; received in revised form 22 December 2006; accepted 4 January 2007) Available online 12 January 2007 